\title{
CD47 Deficiency in Mice Exacerbates Chronic Fatty Diet-Induced Steatohepatitis Through Its Role in Regulating Hepatic Inflammation and Lipid Metabolism
}

\section{OPEN ACCESS}

Edited by:

Robson Coutinho-Silva,

Federal University of Rio de

Janeiro, Brazl

Reviewed by:

Denise Morais da Fonseca,

University of São Paulo, Brazil

Cassiano Felippe

Gonçalves-de-Albuquerque, Universidade Federal do Estado do

Rio de Janeiro, Brazi

*Correspondence:

Ke-Xin Chen

chenkexin@jlu.edu.cn

Yang Zheng

zhengyang@jlu.edu.cn

Yong-Guang Yang

yy2324@columbia.edu

Specialty section: This article was submitted to Inflammation,

a section of the journal

Frontiers in Immunology

Received: 02 October 2019

Accepted: 20 January 2020

Published: 25 February 2020

Citation:

Tao H-C, Chen $K-X$, Wang $X$, Chen $B$, Zhao $W-O$, Zheng $Y$ and Yang $Y-G$

(2020) CD47 Deficiency in Mice

Exacerbates Chronic Fatty Diet-Induced Steatohepatitis Through Its Role in Regulating Hepatic Inflammation and Lipid Metabolism. Front. Immunol. 11:148.

doi: 10.3389/fimmu.2020.00148

\section{Hui-Chao Tao ${ }^{1,2,3}$, Ke-Xin Chen ${ }^{1,2,3 *}$, Xue Wang ${ }^{1,2}$, Bo Chen ${ }^{1,2}$, Wai-Ou Zhao ${ }^{3}$, Yang Zheng ${ }^{3 *}$ and Yong-Guang Yang ${ }^{1,2,4 *}$}

\begin{abstract}
${ }^{1}$ Key Laboratory of Organ Regeneration and Transplantation of the Ministry of Education, Institute of Immunology, The First Hospital, Jilin University, Changchun, China, ${ }^{2}$ National-Local Joint Engineering Laboratory of Animal Models for Human Diseases, Changchun, China, ${ }^{3}$ Cardiovascular Center, The First Hospital, Jilin University, Changchun, China, ${ }^{4}$ Columbia Center for Translational Immunology, Department of Medicine, Columbia University College of Physicians and Surgeons, New York, NY, United States
\end{abstract}

Inflammation is one of the hallmarks of non-alcoholic steatohepatitis. CD47 is a widely expressed transmembrane protein that signals through inhibitory receptor signal regulatory protein $\alpha(\mathrm{SIRP} \alpha)$ to inhibit macrophage activation and phagocytosis. In this study, we sought to investigate the role of CD47 in hepatosteatosis and fibrosis induced by a chronic high-fat diet (HFD), by comparing disease development in wild-type (WT) and CD47KO mice fed HFD for 40 weeks. The HFD induced remarkably more severe hepatic steatosis and fibrosis but less body weight gain and less subcutaneous fat accumulation in CD47KO mice compared to WT mice. Liver tissues from HFD-fed CD47KO mice exhibited enhanced inflammation characterized by increased proinflammatory cytokine production and increased nuclear factor- $\kappa \mathrm{B}$ (NF-kB) activation compared to similarly fed WT mice. Although higher expression of apolipoproteins was observed in CD47KO mice compared to WT mice under a low-fat diet (LFD), HFD-fed WT and CD47KO mice showed comparably prominent downregulation of these apolipoprotein genes, suggesting that the marked difference observed in lipid accumulation and hepatosteatosis between these mice cannot be explained by changes in apolipoproteins. Like apolipoproteins, sirtuin 1 (SIRT1) and peroxisome proliferator activated receptor alpha (PPAR $\alpha$ ), which are involved in regulation of both lipid metabolism and inflammation, were more highly expressed in CD47KO than WT mice under LFD but more severely suppressed in CD47KO than in WT mice under HFD. Taken together, our results indicate that CD47 plays a significant role in the pathogenesis of HFD-induced hepatosteatosis and fibrosis through its role in regulation of inflammation and lipid metabolism.

Keywords: non-alcoholic steatohepatitis-NASH, lipid metabolism, inflammation, cd47, fatty diet 


\section{INTRODUCTION}

Non-alcoholic steatohepatitis (NASH) is the severe form of non-alcoholic fatty liver disease (NAFLD) characterized by excessive fat accumulation and inflammation in the liver $(1,2)$. The pathological features of NASH include hepatic injury, inflammation, and excessive production of extracellular matrix (ECM) proteins, particularly collagen. Activation of proinflammatory factors, such as the transcription factor

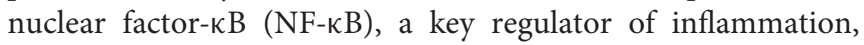
plays an important role in the progression of NASH (3). Although the mechanism for linking inflammation and fibrosis has not yet been fully clarified, liver damage-induced activation of resident inflammatory cells and recruitment of monocytes/macrophages have been reported to promote the development of fibrosis (4). There is increasing evidence to indicate that monocyte/macrophage infiltration results in elevated chemokine production and fibrosis-associated angiogenesis and that increased levels of chemokine C-C motif ligand 2 (CCL2) and its receptor (CCR2) in turn further aggravate monocyte/macrophage infiltration to the site of injury (5-7).

CD47 is a ubiquitously expressed transmembrane protein that serves as a ligand for signal regulatory protein $\alpha(\operatorname{SIRP} \alpha)$ and as a signaling receptor for thrombospondin 1 (TSP1) (8). CD47-SIRP $\alpha$ pathway activation provides inhibitory signaling in monocytes and macrophages, attenuating phagocytosis and inflammation (9-11). This pathway may become even more important in controlling macrophage activation under inflammatory conditions (12) and after allogeneic or xenogeneic transplantation (10,13-15). Although the role of CD47-SIRP $\alpha$ signaling in inhibition of inflammatory responses has been well-documented in numerous models, the involvement of this pathway in fatty acid-induced inflammatory responses is relatively unexplored. A recent report shows that $\mathrm{CD} 47$ deficiency may attenuate obesity and lipid accumulation in the liver of mice fed a high-fat diet (HFD) for 16 weeks (16). Because inflammation is a key factor driving the progression of NASH, it is imperative to understand the precise role of $\mathrm{CD} 47$ in the pathogenesis of NASH.

In this study, we sought to investigate the role of $\mathrm{CD} 47$ in fatty diet consumption-induced NASH by comparing hepatic lipid metabolism, steatosis, fibrosis, and inflammation between wild-type (WT) and CD47KO mice chronically fed HFD for 40 weeks. We show that $\mathrm{CD} 47$ deficiency significantly attenuated body weight gains and subcutaneous fat accumulation, while severely worsening hepatic steatosis and fibrosis induced by prolonged HFD consumption. CD47 deficiency also elevated monocyte/macrophage infiltration, inflammation, and NF- $\kappa \mathrm{B}$ activation in the liver of mice chronically fed HFD. Furthermore, HFD feeding of CD47KO mice induced more significant downregulation of sirtuin 1 (SIRT1) and peroxisome proliferator activated receptor alpha (PPAR $\alpha)$, which are involved in regulation of both lipid metabolism and inflammation. These results provide direct evidence for a significant role of CD47 in regulation of hepatic inflammation and lipid metabolism and in the development of NASH induced by chronic HFD consumption.

\section{METHODS}

\section{Animal Experiments}

WT C57BL/6 (B6) mice and CD47KO mice on the B6 background were originally purchased from Charles River (Beijing, China) and the Jackson laboratory, respectively, and were bred in our specific pathogen-free (SPF) mouse facility for more than a year before use in this study. Eight-weekold male mice were fed HFD (45\% kcal from fat; D12451, XieTong Pharmaceutical Bio-Engineering, China; Table S1) or a low-fat diet (LFD) (10\% kcal from fat; D12450B; XieTong Pharmaceutical Bio-Engineering, China) for 40 weeks. Body weight was measured monthly at the same time. At the indicated times, mice were sacrificed, and blood and liver tissues were collected for analysis as detailed below. Protocols involving animal experiments were approved by the Subcommittee on Research Animal Care of the First Hospital of Jilin University, and all of the experiments were performed in accordance with the protocols.

\section{Analysis of Serum Chemistry}

Mouse serum lipids, including high-density lipoprotein cholesterol (HDL-C), low-density lipoprotein cholesterol (LDL$\mathrm{C})$, and total cholesterol (TC) were examined by Cholesterol E kit (439-17501, Wako) and HDL-Cholesterol E kit (43152501, Wako). Serum alanine aminotransferase (ALT) levels were measured by an ELISA kit for ALT with triplicates for each sample (SEA207Mu, USCN, China) according to the manufacturer's instructions.

\section{Analysis of Lipid Accumulation in Liver}

Liver triglyceride (TG) content was measured using a Triglyceride Assay Kit (Abcam, ab65336) according to the manufacturer's instructions. Lipid droplets were assessed by Oil Red $\mathrm{O}$ staining. Fresh frozen liver tissues were cryostat-sectioned at $5 \mu \mathrm{m}$ and fixed in $4 \%$ PFA for $10 \mathrm{~min}$ at room temperature (RT). Slides were blotted in 60\% isopropyl alcohol for $10 \mathrm{~min}$ and then stained with filtered Oil Red O for 20 min at RT. Slides were rinsed once with distilled water, mounted, and sealed with nail polish.

\section{Quantitative Real-Time PCR}

Total RNA was extracted with an Axyprep multisource RNA miniprep kit (Axygen, America), and cDNA was synthesized using TransScript First-Strand cDNA Synthesis SuperMix (TransGen Biotech, Beijing). Quantitative real-time PCR was performed using a SYBR Green kit (TransGen Biotech, Beijing) with a StepOnePlus real-time PCR system (ABI), and the primer sets used for MTTP, ApoA1, ApoB, ApoC2, CD31, TGF $\beta$, TSP1, VEGFR1, IL-1 $\beta$, TNF $\alpha$, IL-6, IL-10, CCL2, PPAR $\alpha$, SIRT1, and $\beta$-actin (Sangon Biotech, Shanghai) are listed in Table 1. Relative gene expression was measured with triplicates for each sample and normalized to $\beta$-actin. 
TABLE 1 | Primer sequences for qPCR.

\begin{tabular}{|c|c|c|}
\hline $\begin{array}{l}\text { Mouse } \\
\text { genes }\end{array}$ & Forward $\left(5^{\prime}-3^{\prime}\right)$ & Reverse $\left(5^{\prime}-3^{\prime}\right)$ \\
\hline$\beta$-actin & TTCAACACCCCAGCCATG & CCTCGTAGATGGGCACAGT \\
\hline ApoA1 & CTTGGCACGTATGGCAGCA & CCAGAAGTCCCGAGTCAATGG \\
\hline ApoB & ACGGGCAATGAAGACCACAC & CGGGAGCGACACCATTTACAA \\
\hline ApoC2 & ATGGGGTCTCGGTTCTTCCT & GTCTTCTGGTACAGGTCTITGG \\
\hline CD31 & AGGCTTGCATAGAGCTCCAG & TTCTTGGTTCCAGCTATGG \\
\hline TSP1 & TTGCCAGCGTTGCCA & TCTGCAGCACCCCCTGAA \\
\hline TGF $\beta$ & CCACCTGCAAGACCATCGAC & CTGGCGAGCCTTAGTITGGAC \\
\hline TNF $\alpha$ & GTCCTGCTCTACGTGACGAG & TGCAGATGTACCTTGGAGAGTA \\
\hline MTTP & ATACAAGCTCACGTACTCCACT & TCTCTGTTGACCCGCATITC \\
\hline CCL2 & GAAGGAATGGGTCCAGACAT & ACGGGTCAACTTCACATTCA \\
\hline VEGFR1 & CGGAAGGAAGACAGCTCATC & CTTCACGCGACAGGTGTAGA \\
\hline IL-1 $1 \beta$ & TGGACCTTCCAGGATGAGGACA & GTTCATCTCGGAGCCTGTAGTG \\
\hline IL-6 & ССTCTGGTCTTCTGGAGTACC & АCTCCTTCTGTGACTCCAGC \\
\hline IL-10 & АTAACTGCACCCACTTCCCA & GGGCATCACTTCTACCAGGT \\
\hline PPAR $\alpha$ & TACTGCCGTITCACAAGTGC & AGGTCGTGTTCACAGGTAAGA \\
\hline SIRT1 & ATGACGCTGTGGCAGATTGTT & CCGCAAGGCGAGCATAGAT \\
\hline
\end{tabular}

\section{Immunoblotting}

Total lysate was extracted from liver tissues on ice with radioimmunoprecipitation assay (RIPA) lysis buffer and a protease/phosphatase inhibitor cocktail (Roche Diagnostics). The homogenates were centrifuged at $10,000 \times \mathrm{g}$ for $20 \mathrm{~min}$ at $4^{\circ} \mathrm{C}$. The protein amounts were assessed by the BCA method (Thermo, \#23225). Phosphorylated NF-кB p65 (pNF-кB, Ser563) and $\beta$-actin in the total lysate were detected by anti-phosNF- $\kappa$ B (Affinity, AF5006) and anti- $\beta$-actin (Biolegend, 622102) antibodies, respectively. The proteins were visualized with a Super Signal West Pico-Chemiluminescent Substrate (Tanon, 180-501) according to the manufacturer's protocol.

\section{Histology, Immunohistochemistry, and Immunofluorescence Analysis}

Tissues were harvested and fixed with $10 \%$ formalin and embedded in paraffin. Serial sections $(4 \mu \mathrm{m})$ were prepared and stained with $\mathrm{H} \& \mathrm{E}$ and immunohistochemistry (IHC). For IHC, tissue sections were subjected to antigen retrieval and incubation with primary antibody against CCL2 (BF0556, Affinity), TGF- $\beta$ (AF1027, Affinity), IL-1 $\beta$ (AF5103, Affinity), IL-6 (DF6087, Affinity), or IL-10 (DF6894, Affinity), followed by incubation with a peroxidase-conjugated goat anti-rabbit IgG (KIT-9706, Maixin-Bio) secondary antibody, and the immunoreactivity was detected with an UltraSensitive ${ }^{\mathrm{TM}}$ Streptavidin-Peroxidase Kit (KIT-9710, Mai Xin, China) according to the manufacturer's protocol. The collagen content was assessed by Sirius Red (365548, Sigma). For immunofluorescence (IF), cryosections $(4 \mu \mathrm{m})$ were prepared from freshly frozen liver tissues and incubated with antibodies specific for mouse CD68 (137005, Biolegend), CD31antibody (102432, Biolegend), TIE2 (124008, Biolegend), or phos-NF- $\kappa \mathrm{B}$ (Affinity, AF5006). Photomicrographs were produced using a confocal microscope and corresponding software (Zeiss). Image Pro Plus 6.0 software was used for quantitative analysis.

\section{Statistical Analysis}

All data are presented as mean $\pm \mathrm{SD}$, and the statistical analysis was performed using Prism 6 (GraphPad Software). One-way ANOVA was used for comparing multiple groups, while the $t$ test was used for comparing two groups. Differences with $\mathrm{p}<0.05$ were considered statistically significant.

\section{RESULTS}

\section{HFD Induces Severe Hepatomegaly and Liver Injury but Less Subcutaneous Fat Accumulation in CD47-Deficient Mice}

In order to assess the role of CD47 in the development of fatty liver and obesity, we compared hepatic steatosis and subcutaneous fat accumulation in WT and CD47KO mice that were chronically fed LFD ( $10 \% \mathrm{kcal}$ from fat) or HFD ( $45 \%$ $\mathrm{kcal}$ from fat) for 40 weeks. HFD-fed CD47KO mice showed a significantly delayed and lower increase in body weight compared to similarly HFD-fed WT mice (Figure 1A). When fed LFD, WT and CD47KO mice showed a similar increase in body weight within the first 6 months; then the CD47KO mice stopped gaining weight immediately, which was 1 month earlier than the WT mice (Figure 1A). HFD also induced a significant increase in total serum cholesterol (TC; Figure 1B) and LDL-C (Figure 1D) in both WT and CD47KO mice. However, a significant increase in HDL-C was only detected in HFD-fed WT mice (Figure 1C). Furthermore, HFD induced prominent subcutaneous fat accumulation (Figure 1E) and hepatomegaly (Figure 1F) in WT mice. Surprisingly, HFD-fed CD47KO mice showed almost no subcutaneous fat accumulation but extremely severe hepatomegaly compared to HFD-fed WT mice (Figures 1E,F). In HFD-fed CD47KO mice, the liver/body weight ratio was significantly increased (by over two-fold) compared to mice in all other groups (liver/body weight ratios were comparable among LFD-fed WT and CD47KO mice, and HFD-fed WT mice; Figure 1G). Moreover, HFD also resulted in more severe liver injury in CD47KO mice than in WT mice, as shown by ALT serum activity (Figure 1H). These data indicate that CD47 deficiency augments HFD-induced hepatomegaly and liver injury but suppresses subcutaneous fat accumulation and the associated increase in body weight, suggesting that $\mathrm{CD} 47$ plays an important role in lipid metabolism and/or distribution.

\section{CD47 Deficiency in Mice Promotes HFD-Induced Lipid Accumulation in Liver but Not Downregulation of Hepatic Apolipoproteins}

Histological analysis was performed to determine whether CD47 deficiency may promote the development of fatty liver disease. In 


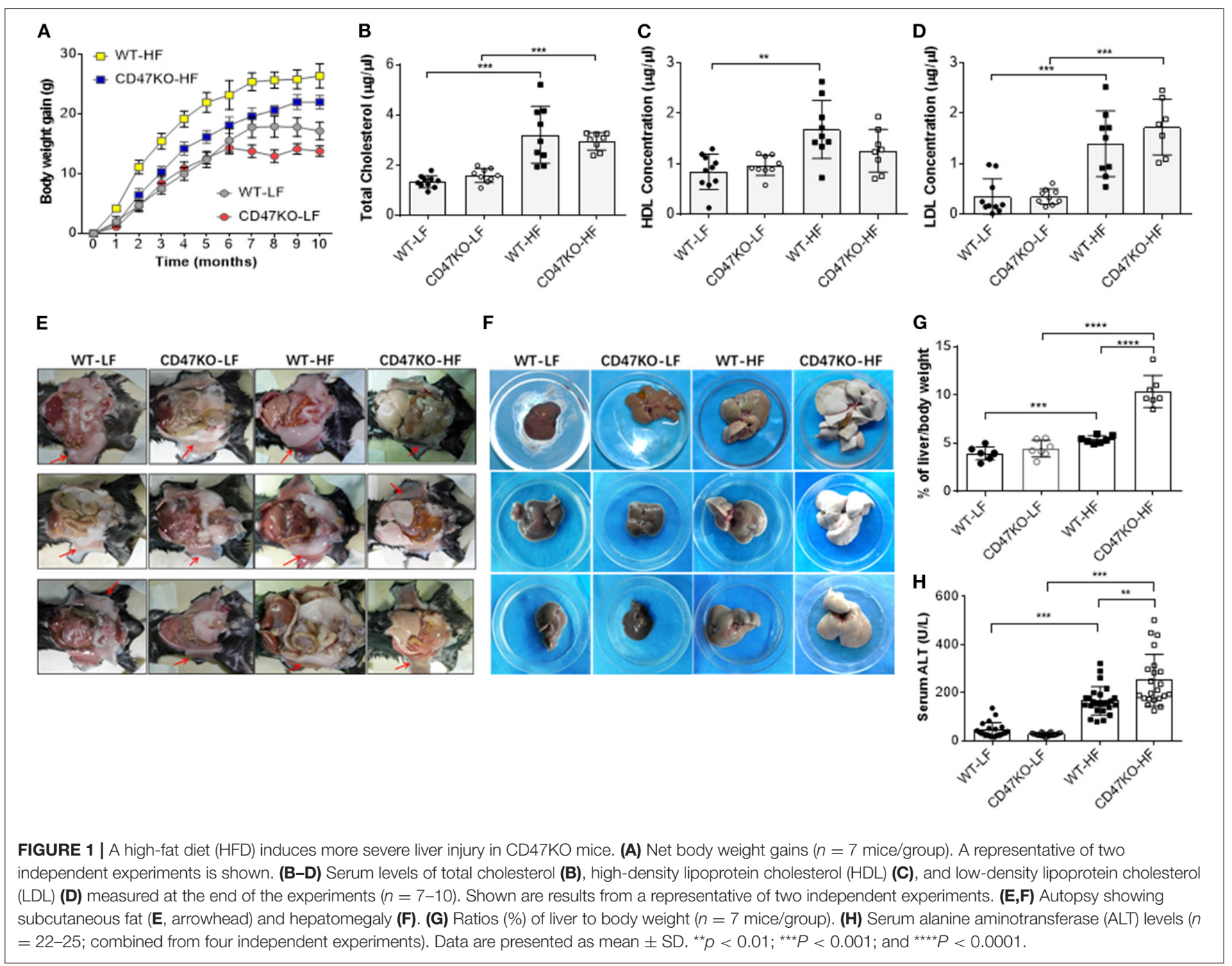

accordance with the more severe hepatomegaly and liver injury in HFD-fed CD47KO mice (Figures 1F-H), H\&E (Figure 2A) and Oil Red $\mathrm{O}$ staining (Figure $2 \mathrm{~B}$ ) revealed that HFD induced more severe hepatocyte ballooning and excessive lipid accumulation in CD47KO mice compared to WT mice, whereas no significant difference was detected between LFD-fed CD47KO and WT mice. Although HFD consistently induced a significant elevation of liver TGs in both WT and CD47KO mice compared to LFD-fed mice, the magnitude of the elevation was significantly less pronounced in WT than CD47KO mice (Figure 2C). Apolipoproteins are involved in the crosstalk between adipose tissue and the liver (17). Because apolipoproteins play important roles in the development of obesity and hepatosteatosis, and their expression can be regulated by liver injury and inflammation (18), we measured the levels of apolipoprotein mRNAs in liver tissues by real-time PCR. Although WT and CD47KO mice fed LFD had comparable expression of APOA1, the levels of APOB, APOC2, and MTTP were significantly higher in the latter group (Figures 2D-G). HFD induced a significant downregulation of all these apolipoproteins in both WT and CD47KO mice (Figures 2D-G). HFD-fed WT and CD47KO mice showed comparably prominent downregulation of these apolipoprotein genes, with the exception of APOC2, which was downregulated to a lesser extent than other apolipoproteins in HFD-fed mice and expressed at a higher level in CD47KO than in WT mice. These data suggest that changes in apolipoproteins cannot explain the observed differences in lipid accumulation and hepatosteatosis between HFD-fed WT and CD47KO mice.

\section{CD47 Deficiency in Mice Promotes Development of HFD-Induced Liver Fibrosis}

Chronic hepatic injury and subsequent inflammation enhance collagen production, resulting in progression of liver fibrosis $(19,20)$. Sirius Red staining revealed that HFD-induced fibrosis, as shown by increased fibrillar collagen deposition, 

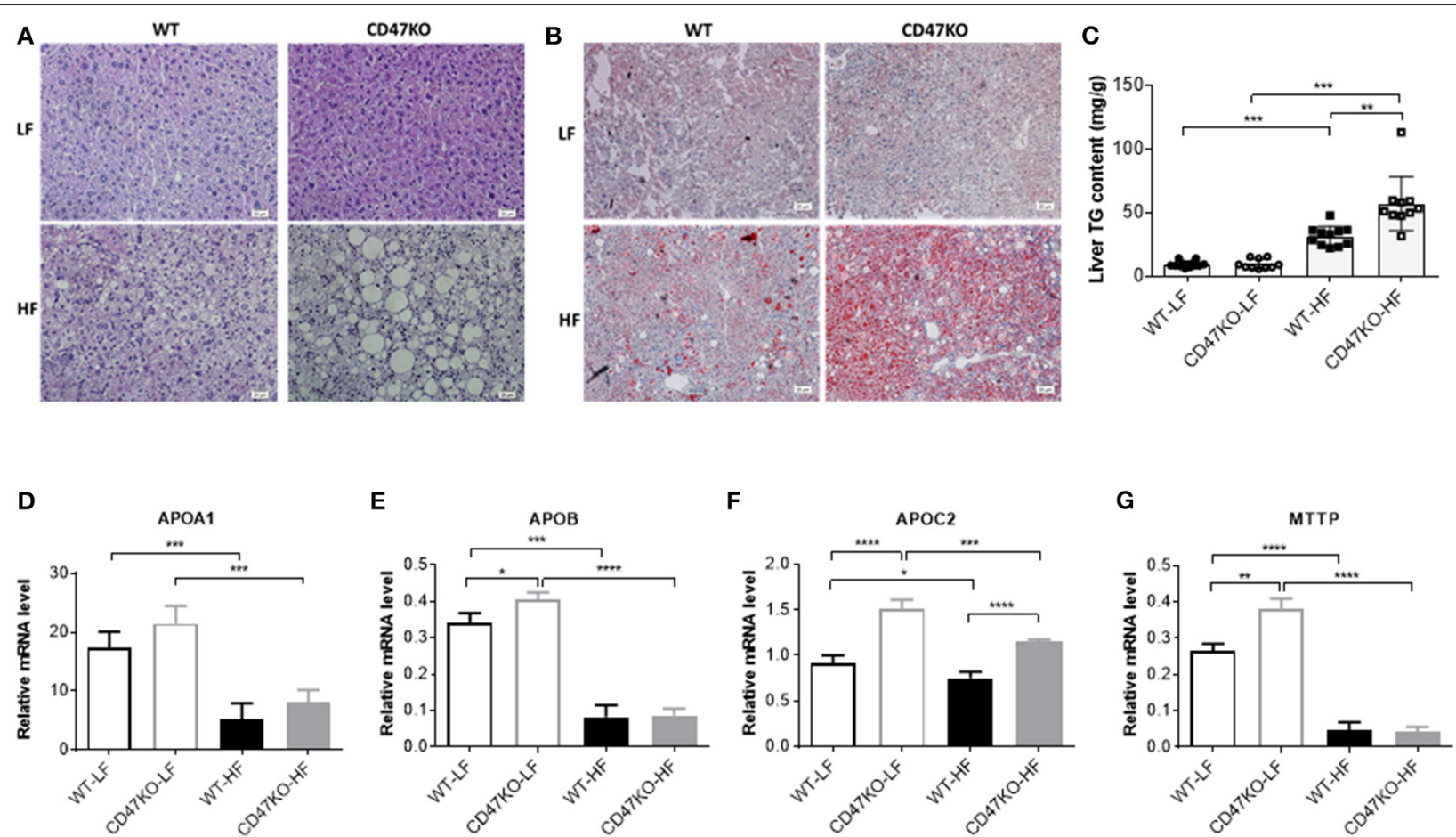

FIGURE 2 | Lipid accumulation and apolipoprotein gene expression in livers from wild-type (WT) and CD47KO mice fed a low-fat diet (LFD) or HFD. (A,B) Representative image of H\&E (A) and Oil Red O (B; lipid droplet accumulation is shown in red) staining of liver tissues from WT and CD47KO mice fed LFD or HFD (three mice per group). Scale bar represents $20 \mu \mathrm{m}$. (C) Triglyceride (TG) content in liver extracts from the indicated groups of mice $(n=10-12$ mice/group; combined from two independent experiments). (D-G) Expression Levels of APOA1 (D), APOB (E), APOC2 (F), and MTTP, which were determined by quantitative real-time PCR and normalized to $\beta$-actin $\left(n=4\right.$ per group). Data are presented as mean $\pm \mathrm{SD}$. ${ }^{\star} p<0.05 ;{ }^{* \star} p<0.01 ;{ }^{* \star \star} P<0.001$; and ${ }^{\star \star \star \star \star} P<0.0001$.

was significantly more severe in CD47KO than in WT mice (Figure 3A). Consistently, liver tissues from HFDfed CD47KO mice displayed significantly higher collagen content (Figure 3B) and upregulated gene expression of pro-fibrotic factor TGF $\beta$ (Figure 3C and Figure S1) and TSP1 (Figure 3D), a major physiologic activator of latent TGFß (8).

\section{HFD Induces Robust Angiogenesis With Upregulation of VEGFR1 and CD31 in the Liver of CD47KO Mice}

Progression of liver fibrosis is accompanied by pathological angiogenesis from the early stages $(21,22)$. To determine the role of CD47 in fibrotic-associated angiogenesis, we measured microvessel density (MVD) by staining with endothelial markers CD31 and TIE2. As shown in Figure 4A, there was a marked increase of MVD in liver tissues from HFDfed CD47KO mice compared to similarly fed WT mice. Furthermore, liver tissues from HFD-fed CD47KO mice also had significantly higher levels of CD31 and VEGFR1 gene expression compared to WT mice (Figures 4A-C). These results indicate that CD47 deficiency may promote HFD-induced collagen production and pro-fibrotic factor expression and thus promote fibrosis and the associated abnormal enhancement of angiogenesis.

\section{CD47 Deficiency in Mice Enhances HFD-Induced Inflammation, Monocyte/Macrophage Infiltration, and NF-кB Activation in the Liver}

Chronic hepatic injury contributes to activation and recruitment of inflammatory cells such as monocytes and macrophages (23). Therefore, we compared the levels of proinflammatory (IL-1 $\beta$, IL-6, and TNF $\alpha$ ) and anti-inflammatory (IL-10) cytokines in liver tissue between WT and CD47KO mice that were chronically fed HFD or LFD. Compared to LFD-fed animals, liver tissues from HFD-fed CD47KO mice showed markedly increased production of all three pro-inflammatory cytokines, whereas only a moderate increase in IL- $1 \beta$ was detected in liver tissues from HFD-fed WT mice (Figures 5A,B,D and Figures S2A,B). However, the level of IL-10 gene expression was significantly higher in liver tissue from HFD-fed WT mice than in liver tissue from HFD-fed CD47KO mice (Figure 5C and Figure S2C). Furthermore, liver tissue from HFD-fed CD47KO mice displayed a more significant increase in production of CCL2 (Figures $5 \mathbf{E}, \mathbf{F}$ ), which was associated with more severe infiltration by CD68+ monocytes/macrophages (Figure 5G), compared to HFD-fed WT mice.

As a master regulator of inflammation, NF- $\mathrm{B}$ activation promotes the secretion of inflammatory cytokines and is accompanied by chronic hepatic injury and the development of liver fibrosis (24). Thus, we next compared NF- $\mathrm{B}$ activation 

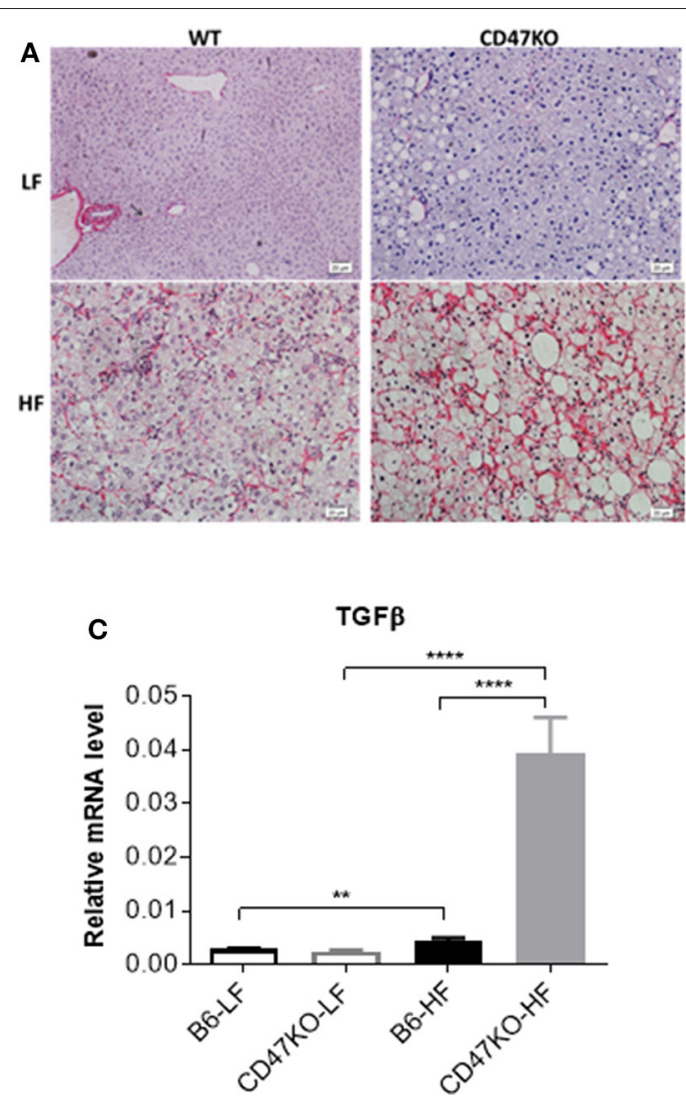
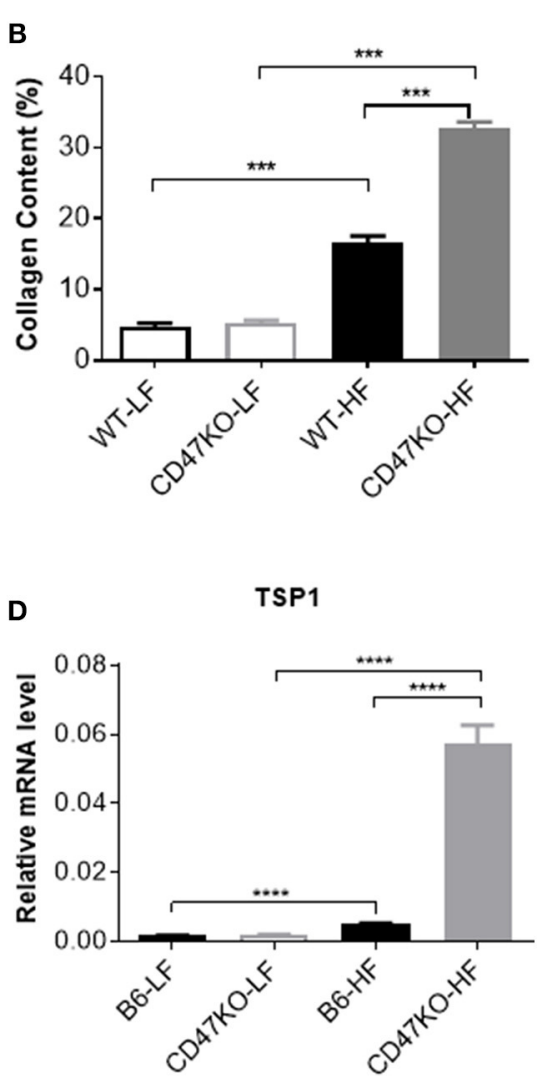

FIGURE 3 | Liver fibrosis in WT and CD47KO mice fed LFD or HFD. (A) Liver fibrosis determined by Sirius Red staining. Three samples per group were examined, and representative images are shown. (B) Collagen content determined by counting positive areas in six randomly selected fields using Image Pro Plus 6.0 software. Data from a representative of three independent samples are shown. (C,D) Relative mRNA expression levels of TGF $\beta$ (C) and thrombospondin 1 (TSP1) (D), which were determined by quantitative real-time PCR and normalized to $\beta$-actin ( $n=4$ per group). Data are presented as mean \pm SD. ${ }^{* \star} p<0.01$; ${ }^{\star \star \star} P<0.001$; and ${ }^{\star \star \star \star} P<0.0001$.

in liver tissues from $\mathrm{WT}$ and $\mathrm{CD} 47 \mathrm{KO}$ mice by measuring phosphorylation at Ser536 and nuclear translocation of NF$\kappa \mathrm{B}$ p65. Although HFD resulted in enhanced NF- $\mathrm{B}$ p65 phosphorylation at Ser536 in both WT and CD47KO mice, the effect was significantly stronger in the latter group (Figure 6A). Furthermore, markedly increased accumulation of phosphorylated NF- $\kappa$ B p65 (Ser536) in the nucleus was detected in liver tissues from HFD-fed CD47KO mice (Figure 6B). Taken together, our results indicate that HFD induces more severe inflammatory responses in CD47KO than in WT mice.

\section{PPAR $\alpha$ and SIRT1 Expression Increases Under LFD Feeding and Is Significantly Downregulated Under HFD Feeding in CD47KO Mice}

We also compared the expression of PPAR $\alpha$ and SIRT1 in liver tissues from WT and CD47KO mice fed LFD or HFD. PPAR $\alpha$, which can be activated by fatty acids, plays an important role in the regulation of hepatic lipid metabolism, and its activity is inversely correlated with NAFLD in both humans and mice (25). PPAR $\alpha$ was also reported to inhibit inflammation via regulation of NF- $\kappa \mathrm{B}$ activity $(26,27)$. SIRT1 inhibits lipogenesis through deacetylating and activating PPAR $\alpha$ (28). Under LFD, significantly increased PPAR $\alpha$ expression was seen in CD47KO mice compared to WT mice (Figure 7A). However, HFD-fed CD47KO mice showed significant downregulation of $\operatorname{PPAR} \alpha$ compared to LFD-fed CD47KO mice, while there was no significant downregulation of PPAR $\alpha$ in HFD-fed WT mice compared to LFD-fed WT mice (Figure 7A). In line with this finding, SIRT1 expression in the liver was also significantly higher under LFD but more severely suppressed by HFD in CD47KO mice compared to WT mice (Figure 7B). These results indicate that CD47 deficiency may affect lipid metabolism under both LFD and HFD conditions. Since PPAR $\alpha$ and SIRT1 also have anti-inflammatory activity, the more severely downregulated expression may also contribute to enhanced hepatic inflammation in HFD-fed CD47KO mice.

\section{DISCUSSION}

NASH involves a range of pathologic conditions in the liver, including steatosis, inflammation, hepatocyte injury, and fibrosis. In this study, we showed that CD47KO mice chronically fed HFD (for 40 weeks) developed severe hepatomegaly and hepatic 

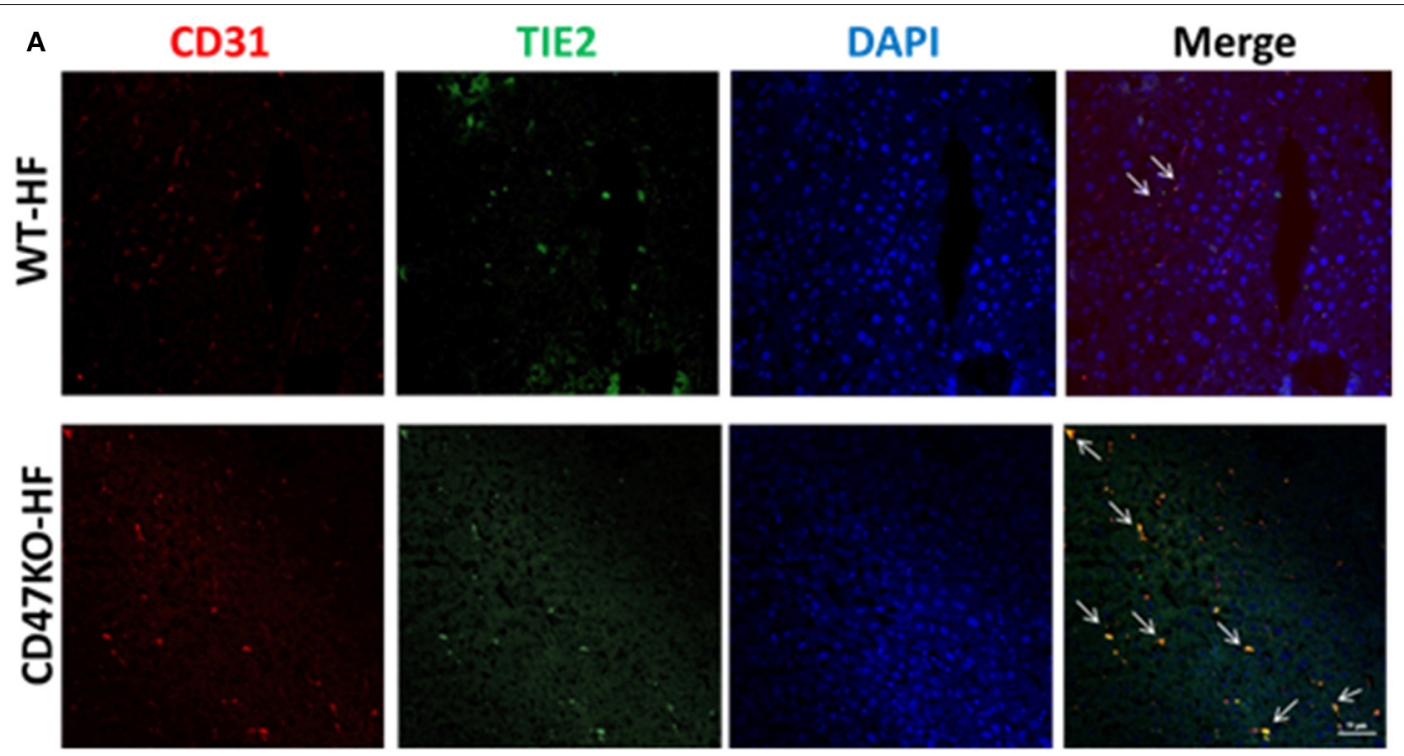

B

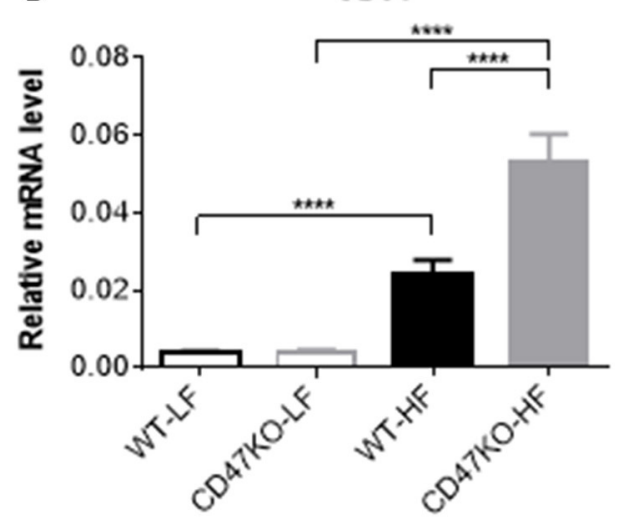

C

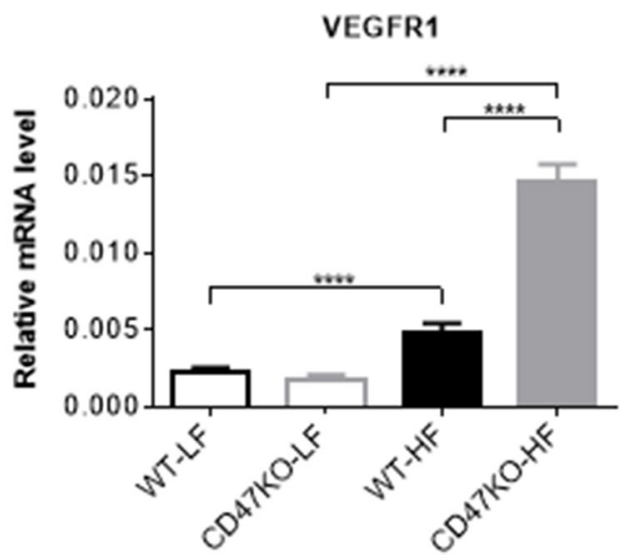

FIGURE 4 | CD47 deficiency promotes HFD-induced angiogenesis with upregulation of CD31 and VEGFR in the liver. (A) Representative images of liver sections stained for CD31 (red), TIE2 (green), and DAPI. Merged images are shown in the last column (CD31+TIE2+ cells are indicated by arrows). Data from a representative of three samples are shown. Scale bar represents $50 \mu \mathrm{m}$. (B,C) Relative mRNA expression levels of CD31 (B) and VEGFR1 (C) quantified by real-time PCR and normalized to $\beta$-actin ( $n=4$ per group). Data are presented as mean $\pm \mathrm{SD}$. ${ }^{\star \star \star \star} P<0.0001$.

injury but no subcutaneous fat accumulation compared to WT mice similarly fed HFD. Liver tissues from HFD-fed CD47KO mice showed significantly more lipid accumulation than liver tissues from HFD-fed WT mice, but both groups had comparable downregulation of apolipoproteins. In livers from HFD-fed CD47KO mice, we consistently found more pronounced collagen production and fibrosis, and the associated abnormally enhanced angiogenesis, compared to HFD-fed WT mice. Notably, HFDfed CD47KO mice exhibited markedly greater proinflammatory responses and more significantly reduced expression of PPAR $\alpha$ and SIRT1, which are both known to regulate lipid metabolism and inflammation (29-32), compared to similarly fed WT mice. These findings provide direct evidence for an important role of CD47 in the pathogenesis of NASH induced by a fatty diet.

Hepatic fibrosis is triggered by chronic liver injury following sustained inflammation. CD47 has been shown to play an important role in regulating macrophage activation and phagocytosis by interacting with $\operatorname{SIRP} \alpha$, an inhibitory receptor on innate immune cells. It has been shown that $\mathrm{CD} 47 / \mathrm{SIRP} \alpha$ is essential in controlling macrophage activation by proinflammatory cytokines (12) and allogeneic or xenogeneic cell stimuli $(10,13-15)$. Here we show that CD47 deficiency also promotes HFD-induced inflammatory responses in the liver, including enhanced proinflammatory cytokine production and the associated activation of NF- $\kappa \mathrm{B}$ and secretion of the potent macrophage chemoattractant chemokine CCL2. Furthermore, increased HFD-induced inflammation in CD47KO mice was correlated with the severity of hepatosteatosis and fibrosis, supporting the previously noted role of inflammation in the development of NAFLD.

In the present study, we found that PPAR $\alpha$ and SIRT1 expression levels in the liver of CD47KO mice were significantly 


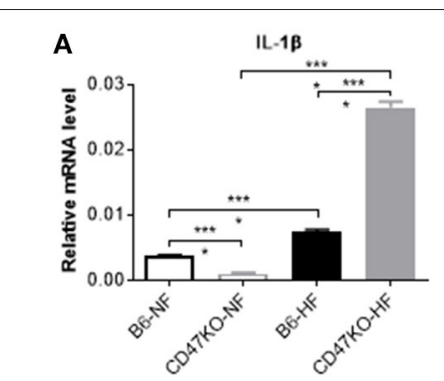

$\mathbf{E}$

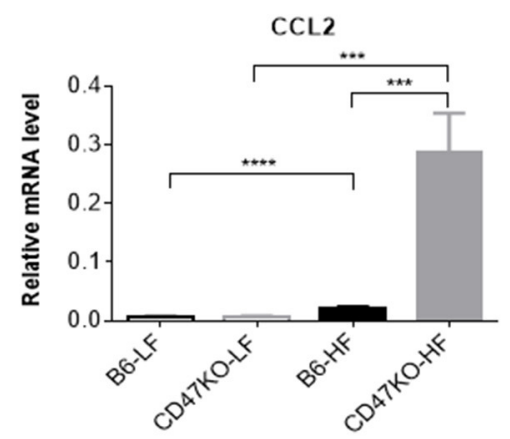

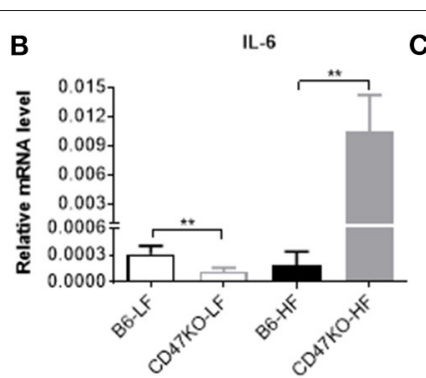

$\mathbf{F}$

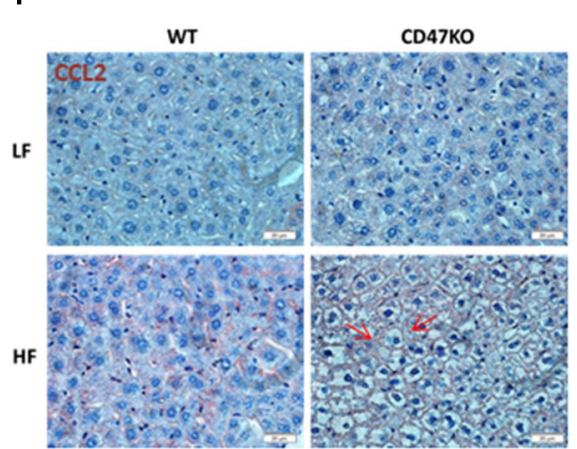

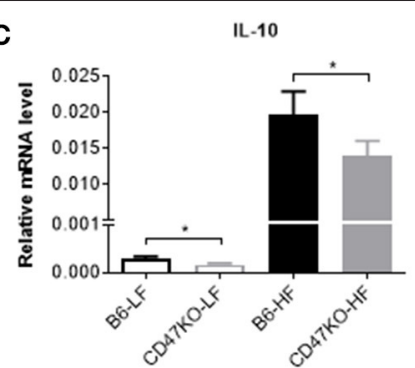

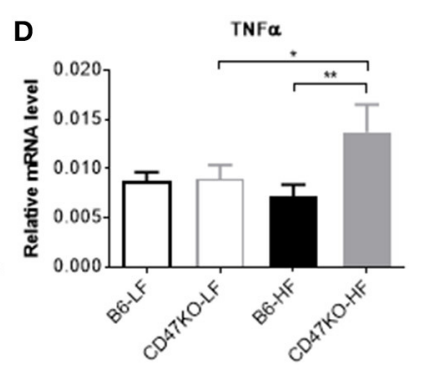

G
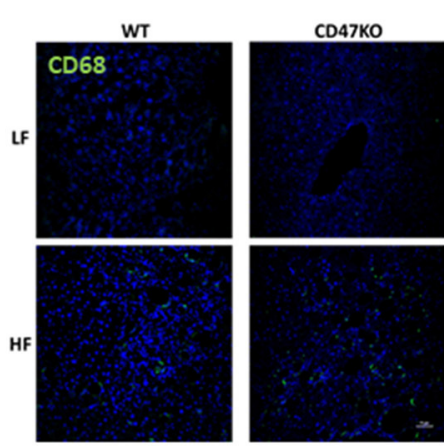

FIGURE 5 | CD47 deficiency enhances HFD-induced inflammation and monocyte/macrophage infiltration in the liver. (A-E) Relative mRNA levels of IL-1 $\beta$ (A), IL-6 (B), IL-10 (C), TNF $\alpha$ (D), and C-C motif ligand 2 (CCL2) (E). Gene expression levels were determined by quantitative real-time PCR and normalized to $\beta$-actin ( $n=4$ per group). Data are presented as mean $\pm \mathrm{SD}$. ${ }^{*} P<0.05$; ${ }^{\star \star} P<0.01$; ${ }^{\star \star *} P<0.001$; and ${ }^{\star \star \star *} P<0.0001$. (F) Liver sections were immunohistochemistry (IHC)-stained for CCL2. Six samples per group were examined, and representative images are shown (scale bar represents $20 \mu \mathrm{m}$ ). (G) Liver sections were immunofluorescence-stained by anti-CD68 (green) plus DAPI (blue). Four samples per group were examined, and representative images are shown (scale bar represents $50 \mu \mathrm{m})$.
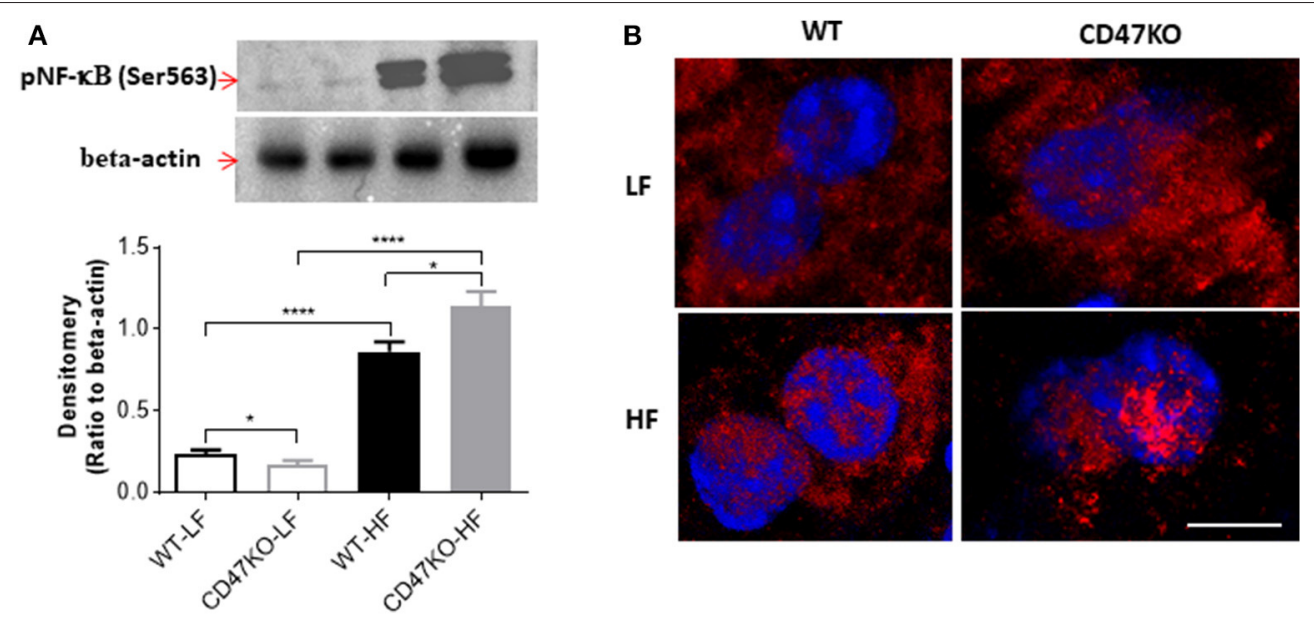

FIGURE 6 | CD47 deficiency promotes HFD-induced nuclear factor-kB (NF-kB) activation and translocation. (A) Immunoblot analysis of phospho-NF-kB p65 (Ser536) protein in liver tissues from WT and CD47KO mice fed LFD or HFD (upper) and band density normalized to $\beta$-actin [lower; data are means ( \pm SDs) of three independent experiments]. (B) Confocal images showing subcellular distribution of phosphorylated NF-kB (pNF-kB) (Ser536); pNF-kB (Ser536); and nucleus were stained in red by AF594 and in blue by DAPI, respectively. The representative images from four independent samples are shown. Scale bar represents $10 \mu \mathrm{m}$. Data are presented as mean \pm SD. ${ }^{*} p<0.05$ and ${ }^{* \star \star *} P<0.0001$.

elevated and inhibited under LFD and HFD conditions, respectively, compared to WT mice. Previous studies have shown that fatty acids play an important role in the activation and stimulation of PPAR $\alpha$ and SIRT1 expression, but NAFLD is often associated with failed/reduced expression of these genes in both humans and mice $(28,33)$. Thus, it is possible that 

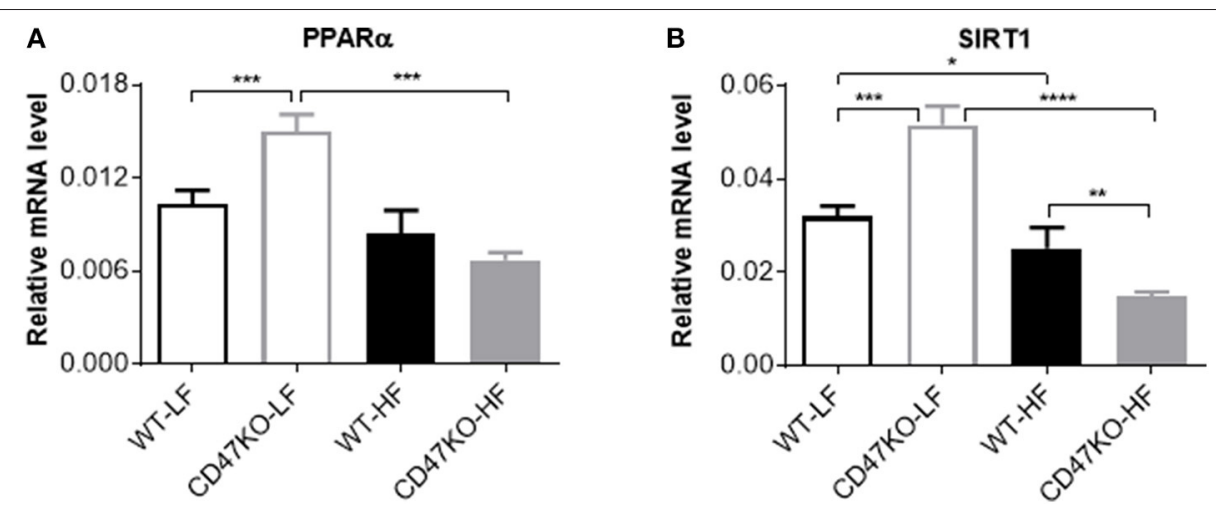

FIGURE 7 | Distinct effects of CD47 deficiency on peroxisome proliferator activated receptor alpha (PPAR $\alpha$ and sirtuin 1 (SIRT1) expression between mice fed LFD vs. HFD. Relative mRNA expression levels of PPAR $\alpha$ (A) and SIRT1 (B) were quantified by real-time PCR and normalized to $\beta$-actin $(n=4$ per group). Data are presented as mean \pm SD. ${ }^{\star} P<0.05 ;{ }^{* *} P<0.01 ;{ }^{* \star *} P<0.001$; and ${ }^{* * \star *} P<0.0001$.

the reduced expression in HFD-fed CD47KO mice was due to severe steatosis similar to that found in patients with established NAFLD. Although further studies are needed to unveil the mechanisms behind the altered expression of PPAR $\alpha$ and SIRT1 in the absence of CD47, our results suggest that CD47 may also be involved in the pathogenesis of HFD-induced NASH through its role in regulating lipid metabolism. In addition to being potent regulators of lipid metabolism, PPAR $\alpha$ and SIRT1 have also been reported to inhibit proinflammatory responses. SIRT1 has a pivotal role in the regulation of PPAR $\alpha$ activation and antagonistic inhibition of NF- $\kappa \mathrm{B}$ in the liver $(34,35)$. Furthermore, PPAR $\alpha$ activation downregulates IL-1 $\beta$ and $\mathrm{TNF} \alpha$ in the liver via inhibition of NF- $\mathrm{B}$ and suppresses hepatosteatosis via upregulation of fatty acid oxidation gene expression in rodent NAFLD models $(31,32)$. Here we found that the levels of PPAR $\alpha$ and SIRT1 expression were inversely correlated with NF- $\mathrm{B}$ activity in HFD-fed mice, which is consistent with the previously reported antagonistic crosstalk between NF- $\kappa$ B and SIRT1 $(36,37)$. Taken together, these observations indicate that the more severe inhibition of PPAR $\alpha$ and SIRT1 expression may contribute to the augmented hepatic inflammation in HFD-fed CD47KO mice.

HFD induces a marked increase in circulating TSP1 in the early stages of HFD challenge, and TSP1 deficiency protects mice from HFD-induced weight gain and adipocyte hypertrophy (38). Thus, it is likely that the loss of TSP1/CD47 signaling also contributes to the reduced weight gain and reduced subcutaneous fat accumulation observed in $\mathrm{CD} 47 \mathrm{KO}$ mice fed HFD. However, the current study cannot mechanistically explain the contradictory findings in subcutaneous vs. liver fat metabolism in CD47KO mice under HFD. A previous report showed that CD47 deficiency protects against fat accumulation in both subcutaneous tissue and liver, in mice fed HFD for a shorter period of time (16 weeks) (16). It is possible that the effect of CD47 deficiency may differ depending on the conditions of the tissue microenvironment. These differences might include the proclivity of tissue-resident cells to cause and/or respond to inflammation and the strength of proinflammatory stimuli, and the degree of tissue damage. These factors are expected to be different depending on the tissue and the time after HFD feeding.

TSP1 is a potent inhibitor of angiogenesis (39), and its anti-angiogenic activity can be mediated by its receptor CD47 (40-42). In line with these observations, CD47 deficiency in endothelial cells (ECs) was found to enable ECs to gain stem cell characteristics (43), and to improve angiogenic function by promoting proliferation and attenuating replicative senescence of ECs (40). TSP1/CD47 signaling also inhibits VEGF2 phosphorylation in ECs (44), and CD47 deficiency enhances neovascularization in tumors (45). Thus, abrogation of TSP1/CD47 signaling is likely to be a key mechanism for the observed upregulation of VEGFR and the abnormally enhanced angiogenesis in the livers of HFD-fed CD47KO mice.

In conclusion, this study provides direct evidence that CD47, a ligand of $\operatorname{SIRP} \alpha$, an inhibitory receptor on myeloid innate immune cells, plays a significant role in the pathogenesis of HFD-induced hepatosteatosis and fibrosis. Our data also suggest that the role of CD47 in NASH induced by chronic HFD consumption is mediated by its effects on hepatic inflammation and lipid metabolism.

\section{DATA AVAILABILITY STATEMENT}

The datasets generated for this study are available on request to the corresponding author.

\section{ETHICS STATEMENT}

This animal study was reviewed and approved by Subcommittee on Research Animal Care of the First Hospital of Jilin University.

\section{AUTHOR CONTRIBUTIONS}

$\mathrm{H}-\mathrm{CT}$ and $\mathrm{K}-\mathrm{XC}$ designed, performed, and analyzed data from most of the experiments. XW, BC, and W-OZ performed histology and immunofluorescence analysis. K-XC, YZ, and 
Y-GY conceived, designed, and supervised all studies. K-XC and Y-GY wrote the manuscript. All authors read and approved the final manuscript.

\section{FUNDING}

This work was supported by grants from the Chinese MOST (2015CB964400) and NSFC (91642208, 81273334, and 81302611).

\section{REFERENCES}

1. Carr RM, Oranu A, Khungar V. Nonalcoholic fatty liver disease: pathophysiology and management. Gastroenterol Clin North Am. (2016) 45:639-52. doi: 10.1016/j.gtc.2016.07.003

2. Liu W, Baker RD, Bhatia T, Zhu L, Baker SS. Pathogenesis of nonalcoholic steatohepatitis. Cell Mol Life Sci. (2016) 73:1969-87. doi: 10.1007/s00018-016-2161-x

3. Luedde T, Beraza N, Kotsikoris V, van Loo G, Nenci A, De Vos R, et al. Deletion of NEMO/IKKgamma in liver parenchymal cells causes steatohepatitis and hepatocellular carcinoma. Cancer Cell. (2007) 11:119-32. doi: 10.1016/j.ccr.2006.12.016

4. Hernandez-Gea V, Friedman SL. Pathogenesis of liver fibrosis. Annu Rev Pathol. (2011) 6:425-56. doi: 10.1146/annurev-pathol-011110-130246

5. Ehling J, Bartneck M, Wei X, Gremse F, Fech V, Mockel D, et al. CCL2dependent infiltrating macrophages promote angiogenesis in progressive liver fibrosis. Gut. (2014) 63:1960-71. doi: 10.1136/gutjnl-2013-306294

6. Zhang J, Xu P, Song P, Wang H, Zhang Y, Hu Q, et al. CCL2-CCR2 signaling promotes hepatic ischemia/reperfusion injury. J Surg Res. (2016) 202:352-62. doi: 10.1016/j.jss.2016.02.029

7. Baeck C, Wehr A, Karlmark KR, Heymann F, Vucur M, Gassler N, et al. Pharmacological inhibition of the chemokine CCL2 (MCP-1) diminishes liver macrophage infiltration and steatohepatitis in chronic hepatic injury. Gut. (2012) 61:416-26. doi: 10.1136/gutjnl-2011-300304

8. Brown EJ, Frazier WA. Integrin-associated protein (CD47) and its ligands. Trends Cell Biol. (2001) 11:130-5. doi: 10.1016/S0962-8924(00) 01906-1

9. Matozaki T, Murata Y, Okazawa H, Ohnishi H. Functions and molecular mechanisms of the CD47-SIRPalpha signalling pathway. Trends Cell Biol. (2009) 19:72-80. doi: 10.1016/j.tcb.2008.12.001

10. Wang H, Wu X, Wang Y, Oldenborg PA, Yang YG. CD47 is required for suppression of allograft rejection by donor-specific transfusion. I Immunol. (2010) 184:3401-7. doi: 10.4049/jimmunol.0901550

11. Veillette A, Chen J. SIRPalpha-CD47 Immune Checkpoint Blockade in Anticancer Therapy. Trends Immunol. (2018) 39:173-84. doi: 10.1016/j.it.2017.12.005

12. Bian Z, Shi L, Guo YL, Lv Z, Tang C, Niu S, et al. Cd47-Sirpalpha interaction and IL-10 constrain inflammation-induced macrophage phagocytosis of healthy self-cells. Proc Natl Acad Sci USA. (2016) 113:E5434-43. doi: 10.1073/pnas.1521069113

13. Ide K, Wang H, Tahara H, Liu J, Wang X, Asahara T, et al. Role for CD47SIRPalpha signaling in xenograft rejection by macrophages. Proc Natl Acad Sci USA. (2007) 104:5062-6. doi: 10.1073/pnas.0609661104

14. Wang H, VerHalen J, Madariaga ML, Xiang S, Wang S, Lan P, et al. Attenuation of phagocytosis of xenogeneic cells by manipulating CD47. Blood. (2007) 109:836-42. doi: 10.1182/blood-2006-04-019794

15. Zhang $M$, Wang $H$, Tan S, Navarro-Alvarez N, Zheng $Y$, Yang YG. Donor CD47 controls $\mathrm{T}$ cell alloresponses and is required for tolerance induction following hepatocyte allotransplantation. Sci Rep. (2016) 6:26839. doi: 10.1038/srep26839

16. Maimaitiyiming H, Norman H, Zhou Q, Wang S. CD47 deficiency protects mice from diet-induced obesity and improves whole body glucose tolerance and insulin sensitivity. Sci Rep. (2015) 5:8846. doi: 10.1038/srep08846

\section{ACKNOWLEDGMENTS}

The authors thank Ms. Meifang Wang and Mr. Zhanwei Sun for their excellent animal care.

\section{SUPPLEMENTARY MATERIAL}

The Supplementary Material for this article can be found online at: https://www.frontiersin.org/articles/10.3389/fimmu. 2020.00148/full\#supplementary-material

17. Wu CL, Zhao SP, Yu BL. Intracellular role of exchangeable apolipoproteins in energy homeostasis, obesity and non-alcoholic fatty liver disease. Biol Rev Camb Philos Soc. (2015) 90:367-76. doi: 10.1111/brv.12116

18. Navarro MA, Carpintero R, Acin S, Arbones-Mainar JM, Calleja L, Carnicer R, et al. Immune-regulation of the apolipoprotein A-I/C-III/AIV gene cluster in experimental inflammation. Cytokine. (2005) 31:52-63. doi: 10.1016/j.cyto.2005.03.002

19. Bataller R, Brenner DA. Liver fibrosis. J Clin Invest. (2005) 115:209-18. doi: 10.1172/JCI24282

20. Friedman SL. Mechanisms of hepatic fibrogenesis. Gastroenterology. (2008) 134:1655-69. doi: 10.1053/j.gastro.2008.03.003

21. Corpechot C, Barbu V, Wendum D, Kinnman N, Rey C, Poupon R, et al. Hypoxia-induced VEGF and collagen I expressions are associated with angiogenesis and fibrogenesis in experimental cirrhosis. Hepatology. (2002) 35:1010-21. doi: 10.1053/jhep.2002.32524

22. Medina J, Arroyo AG, Sanchez-Madrid F, Moreno-Otero R. Angiogenesis in chronic inflammatory liver disease. Hepatology. (2004) 39:1185-95. doi: 10.1002/hep.20193

23. Tacke F. Functional role of intrahepatic monocyte subsets for the progression of liver inflammation and liver fibrosis in vivo. Fibrogenesis Tissue Repair. (2012) 5(Suppl. 1):S27. doi: 10.1186/1755-1536-5-S1-S27

24. Luedde T, Schwabe RF. NF-kappaB in the liver-linking injury, fibrosis and hepatocellular carcinoma. Nat Rev Gastroenterol Hepatol. (2011) 8:108-18. doi: $10.1038 /$ nrgastro.2010.213

25. Pawlak M, Lefebvre P, Staels B. Molecular mechanism of PPARalpha action and its impact on lipid metabolism, inflammation and fibrosis in non-alcoholic fatty liver disease. J Hepatol. (2015) 62:720-33. doi: 10.1016/j.jhep.2014.10.039

26. Stienstra R, Mandard S, Tan NS, Wahli W, Trautwein C, Richardson TA, et al. The Interleukin-1 receptor antagonist is a direct target gene of PPARalpha in liver. J Hepatol. (2007) 46:869-77. doi: 10.1016/j.jhep.2006.11.019

27. Kleemann R, Gervois PP, Verschuren L, Staels B, Princen HM, Kooistra T. Fibrates down-regulate IL-1-stimulated C-reactive protein gene expression in hepatocytes by reducing nuclear p50-NFkappa B-C/EBP-beta complex formation. Blood. (2003) 101:545-51. doi: 10.1182/blood-2002-06-1762

28. Purushotham A, Schug TT, Xu Q, Surapureddi S, Guo X, Li X. Hepatocyte-specific deletion of SIRT1 alters fatty acid metabolism and results in hepatic steatosis and inflammation. Cell Metab. (2009) 9:327-38. doi: 10.1016/j.cmet.2009.02.006

29. Yoshizaki T, Milne JC, Imamura T, Schenk S, Sonoda N, Babendure JL, et al. SIRT1 exerts anti-inflammatory effects and improves insulin sensitivity in adipocytes. Mol Cell Biol. (2009) 29:1363-74. doi: 10.1128/MCB.00705-08

30. Gillum MP, Kotas ME, Erion DM, Kursawe R, Chatterjee P, Nead KT, et al. SirT1 regulates adipose tissue inflammation. Diabetes. (2011) 60:3235-45. doi: $10.2337 / \mathrm{db} 11-0616$

31. Gervois P, Kleemann R, Pilon A, Percevault F, Koenig W, Staels B, et al. Global suppression of IL-6-induced acute phase response gene expression after chronic in vivo treatment with the peroxisome proliferator-activated receptor-alpha activator fenofibrate. J Biol Chem. (2004) 279:16154-60. doi: 10.1074/jbc.M400346200

32. Ip E, Farrell GC, Robertson G, Hall P, Kirsch R, Leclercq I. Central role of PPARalpha-dependent hepatic lipid turnover in dietary steatohepatitis in mice. Hepatology. (2003) 38:123-32. doi: 10.1053/jhep.2003.50307 
33. Huang K, Du M, Tan X, Yang L, Li X, Jiang Y, et al. PARP1-mediated PPARalpha poly(ADP-ribosyl)ation suppresses fatty acid oxidation in non-alcoholic fatty liver disease. J Hepatol. (2017) 66:962-77. doi: 10.1016/j.jhep.2016.11.020

34. Bjurberg M, Henriksson E, Brun E, Ekblad L, Ohlsson T, Brun A, et al. Early changes in 2-deoxy-2-[18F]fluoro-D-glucose metabolism in squamouscell carcinoma during chemotherapy in vivo and in vitro. Cancer Biother Radiopharm. (2009) 24:327-32. doi: 10.1089/cbr.2008.0556

35. Xu F, Gao Z, Zhang J, Rivera CA, Yin J, Weng J, et al. Lack of SIRT1 (Mammalian Sirtuin 1) activity leads to liver steatosis in the SIRT1+/- mice: a role of lipid mobilization and inflammation. Endocrinology. (2010) 151:250414. doi: 10.1210/en.2009-1013

36. Yeung F, Hoberg JE, Ramsey CS, Keller MD, Jones DR, Frye RA, et al. Modulation of NF-kappaB-dependent transcription and cell survival by the SIRT1 deacetylase. EMBO J. (2004) 23:2369-80. doi: 10.1038/sj.emboj.7600244

37. Yang XD, Tajkhorshid E, Chen LF. Functional interplay between acetylation and methylation of the RelA subunit of NF-kappaB. Mol Cell Biol. (2010) 30:2170-80. doi: 10.1128/MCB.01343-09

38. Inoue M, Jiang Y, Barnes RH 2nd, Tokunaga M, Martinez-Santibanez G, Geletka L, et al. Thrombospondin 1 mediates high-fat diet-induced muscle fibrosis and insulin resistance in male mice. Endocrinology. (2013) 154:454859. doi: 10.1210/en.2013-1587

39. Zaslavsky A, Baek KH, Lynch RC, Short S, Grillo J, Folkman J, et al. Platelet-derived thrombospondin-1 is a critical negative regulator and potential biomarker of angiogenesis. Blood. (2010) 115:4605-13. doi: 10.1182/blood-2009-09-242065

40. Gao Q, Chen K, Gao L, Zheng Y, Yang YG. Thrombospondin-1 signaling through CD47 inhibits cell cycle progression and induces senescence in endothelial cells. Cell Death Dis. (2016) 7:e2368. doi: 10.1038/cddis.2016.155
41. Isenberg JS, Ridnour LA, Dimitry J, Frazier WA, Wink DA, Roberts DD. CD47 is necessary for inhibition of nitric oxide-stimulated vascular cell responses by thrombospondin-1. J Biol Chem. (2006) 281:26069-80. doi: 10.1074/jbc.M605040200

42. Kanda S, Shono T, Tomasini-Johansson B, Klint P, Saito Y. Role of thrombospondin-1-derived peptide, 4N1K, in FGF-2-induced angiogenesis. Exp Cell Res. (1999) 252:262-72. doi: 10.1006/excr.1999.4622

43. Kaur S, Soto-Pantoja DR, Stein EV, Liu C, Elkahloun AG, Pendrak ML, et al. Thrombospondin-1 signaling through CD47 inhibits self-renewal by regulating c-Myc and other stem cell transcription factors. Sci Rep. (2013) 3:1673. doi: 10.1038/srep01673

44. Kaur S, Martin-Manso G, Pendrak ML, Garfield SH, Isenberg JS, Roberts DD. Thrombospondin-1 inhibits VEGF receptor-2 signaling by disrupting its association with CD47. J Biol Chem. (2010) 285:38923-32. doi: $10.1074 /$ jbc.M110.172304

45. Gao L, Chen K, Gao Q, Wang X, Sun J, Yang YG. CD47 deficiency in tumor stroma promotes tumor progression by enhancing angiogenesis. Oncotarget. (2017) 8:22406-13. doi: 10.18632/oncotarget.9899

Conflict of Interest: The authors declare that the research was conducted in the absence of any commercial or financial relationships that could be construed as a potential conflict of interest.

Copyright (c) 2020 Tao, Chen, Wang, Chen, Zhao, Zheng and Yang. This is an openaccess article distributed under the terms of the Creative Commons Attribution License (CC BY). The use, distribution or reproduction in other forums is permitted, provided the original author(s) and the copyright owner(s) are credited and that the original publication in this journal is cited, in accordance with accepted academic practice. No use, distribution or reproduction is permitted which does not comply with these terms. 